\title{
Integrität und Transparenz im Heilmittelbereich ab 1. Januar 2020
}

\author{
Ursina Pally Hofmann \\ Dr. iur., Rechtsanwältin, Generalsekretärin und Leiterin Rechtsdienst FMH
}

Die per 1. Januar 2020 in Kraft tretende Verordnung über Integrität und Transparenz im Heilmittelbereich regelt die Details der im Heilmittelgesetz in den ebenfalls neu in Kraft tretenden Art. 55 und 56 aufgeführten Bestimmungen [1]. Weiter werden dann auch die neuen Vorschriften im Zusammenhang mit der Weitergabe von Vergünstigungen gemäss Krankenversicherungsverordnung anwendbar sein. Nachfolgend werden die wichtigsten Grundsätze aufgeführt, wobei sich in den Erläuterungen des Eidgenössischen Departements des Innern EDI noch weitere Beispiele und Hinweise finden lassen [2].

In den folgenden Wochen werden in der SÄZ zu diesem Thema weitere Publikationen erfolgen. Unter anderem mehrere Ausgaben mit Beispielen in der Form von Fragen und Antworten.

\section{Integrität}

Alle Personen, die verschreibungspflichtige Arzneimittel selbst verschreiben, abgeben oder eigenverantwortlich beruflich anwenden, sowie diejenigen, welche solche Arzneimittel zur Verschreibung, Abgabe oder beruflichen Anwendung einkaufen oder über deren Einkauf mitentscheiden, gelten als Fachpersonen und haben sich an die Integritätsvorschriften zu halten [3].

Vorteile von bescheidenem Wert, die für die medizinische und pharmazeutische Praxis von Belang sind, gelten nicht als «nicht gebührende Vorteile".

Auch Assistenzärztinnen wenden Arzneimittel eigenverantwortlich an, weshalb sie unter diese Bestimmung fallen [4]. Neben den Fachpersonen sind Organisationen Adressaten dieser Bestimmungen. Diese können allenfalls sogar strafrechtlich für das Handeln ihrer Entscheidungsträger verantwortlich gemacht werden [5]. Als Organisationen im Sinne der Verordnung gelten juristische Personen, welche Fachpersonen beschäftigen [6].

Vorteile von bescheidenem Wert, die für die medizinische und pharmazeutische Praxis von Belang sind, gelten nicht als «nicht gebührende Vorteile». Um als Vor- teil von bescheidenem Wert zu gelten, darf dieser einen jährlichen Betrag von CHF 300 pro Fachperson, wobei dieser Beitrag pro Organisation nur einmal bezahlt werden darf, selbst wenn diese mehrere Fachpersonen beschäftigt, nicht überschreiten [7]. Weiter ist die Ausrichtung dieses Betrags unabhängig von allfälligen Unterstützungsbeiträgen für Weiter- und Fortbildungsveranstaltungen zu bemessen.

Als von Belang für die medizinische und pharmazeutische Praxis gelten Vorteile, welche den Patienten direkt oder indirekt zugutekommen. Letzteres ist gegeben, wenn ein unmittelbarer Bezug zur Berufsausübung der Fachperson besteht, wenn also die Fachperson ihr Fachwissen erweitern kann durch den Einsatz von Fachliteratur, den Besuch von Weiter- oder Fortbildungen mittels elektronischer Medien, oder wenn es um Arbeitsgeräte wie Fiebermesser oder Praxissoftware geht. Nicht dazu gehören Einladungen der Fachperson zu Mittagessen oder persönliche Geschenke an diese.

Ein direkter Patientennutzen besteht dann, wenn die Praxisausstattung verbessert wird, beispielsweise durch die Installation von Wasserspendern, Kinderspielzeug oder Lektüre im Wartezimmer [8].

\section{Gewinne und Preise im Rahmen von Wettbewerben}

Wettbewerbsgewinne sind erlaubt, sofern jeder der ausgeschriebenen Gewinne oder Preise für sich alleine betrachtet bescheiden sowie für die medizinische und pharmazeutische Praxis von Belang ist, und 
sich der Wettbewerb ausschliesslich an ein Fachpublikum richtet. Weiter darf er nicht mit der Bestellung von verschreibungspflichtigen Arzneimitteln verbunden sein [9].

\section{Unterstützung von Forschung, Lehre und Infrastruktur}

Auch die Unterstützung von Forschung gilt unter bestimmten Voraussetzungen nicht als «nicht gebührender Vorteil». Das EDI geht davon aus, dass entgegen dem Gesetzestext auch Unterstützungsbeiträge an Organisationen zulässig sind, sofern sie Lehre und Infrastruktur betreffen [10]. Wird keine direkte Gegenleistung angeboten - handelt es sich also um ein Sponsoring -, dürfen die Unterstützungsbeiträge nicht an einzelne Fachpersonen ausgerichtet oder versprochen, sondern nur Organisationen angeboten, versprochen oder gewährt werden. Weiter wird vorausgesetzt, dass eine schriftliche Vereinbarung, welche den beabsichtigten Verwendungszweck festhält, abgeschlossen wird. Die Beiträge dürfen ausschliesslich zweckgebunden verwendet und nur auf ein dafür bestimmtes Konto der Organisation überwiesen werden. Darauf dürfen Fachpersonen keinen Zugriff haben, und die Beträge müssen in der Buchhaltung der Organisation ausgewiesen sein. Eine Unterstützung darf zudem nicht an Bedingungen und Auflagen geknüpft sein, sofern die Verschreibung, Abgabe, Anwendung oder der Bezug bestimmter verschreibungspflichtiger Arzneimittel davon betroffen ist [11].

\section{Unterstützung von Weiter- und Fortbildung von Fachpersonen}

Unter denselben Voraussetzungen sind Unterstützungsbeiträge für die Weiter- und Fortbildung von Fachpersonen erlaubt, wobei die jeweilige Organisation unabhängig über die Art und Auswahl der Weiteroder Fortbildung sowie über die teilnehmende Fachperson entscheiden können muss [12].

An die Teilnahme von Fachpersonen an Weiterbildungs- und Fortbildungsveranstaltungen dürfen auch persönliche Beiträge ausgerichtet werden. Die Unterstützung muss sich auf Kosten beschränken, welche im Rahmen der Veranstaltung anfallen, weshalb zusätzliche Reisen, Übernachtungen und Zwischenaufenthalte nicht darunter fallen [13].

Zulässig sind Unterstützungsbeiträge, sofern sie vorgängig schriftlich vereinbart wurden und die teilnehmende Person oder die sie beschäftigende Organisation ebenfalls einen angemessenen Beitrag an die
Veranstaltungskosten leistet. Dieser kann in Geld oder in einer anderen Gegenleistung wie beispielsweise einem Referat, der Sitzungsleitung oder einer Posterpräsentation bestehen, wobei die allgemeinen für Gegenleistungen geltenden Regeln eingehalten werden müssen, vgl. dazu die nachfolgenden Ausführungen zu den Gegenleistungen [14].

Der Selbstkostenbeitrag muss sich bei Fortbildungsveranstaltungen mindestens auf einen Drittel, bei Weiterbildungsveranstaltungen auf einen Fünftel der auf die jeweilige Person entfallenden Veranstaltungskosten belaufen. Angerechnet wird der Selbstkostenbeitrag an die Teilnahmegebühren, Hin- und Rückreise, Unterkunft, Verpflegung und Rahmenprogramme von deutlich untergeordneter Bedeutung.

Eine mitreisende Begleitperson hat - auch wenn sie selbst Fachperson ist - ihre Kosten selbst zu tragen.

Alle Angebote einer Fort- und Weiterbildungsveranstaltung, welche für die Teilnahme an der Veranstaltung nicht erforderlich, also von deutlich untergeordneter Bedeutung sind, gelten als Rahmenprogramm, das erlaubt ist. Darunter fallen Begleitanlässe kultureller, sozialer, touristischer und sportlicher sowie kulinarischer Natur, wenn letztere über die erforderliche Verpflegung hinausgehen.

Eine mitreisende Begleitperson hat - auch wenn sie selbst Fachperson ist - ihre Kosten selbst zu tragen, was auch für allfällige durch ihre eigene Teilnahme an der fachlichen Veranstaltung verursachte Kosten gilt [15]. Veranstaltungen, die höchstens einen halben Arbeitstag dauern und weder mit einer anschliessenden Verpflegung noch mit einer Übernachtung vor Ort verbunden sind, sind von der Entrichtung eines Selbstkostenanteils ausgenommen [16].

Nicht erlaubt ist die Umgehung dieser Vorschriften durch eine ganze oder teilweise Rückerstattung des geleisteten Selbstkostenanteils oder die Übernahme von Kosten, die indirekt durch die Teilnahme entstehen, wie Arbeitsausfall oder Betriebskosten [17]

\section{Gleichwertige Gegenleistungen}

Gleichwertige Gegenleistungen, etwa bei der Bestellung und Lieferung von Heilmitteln, gelten nicht als «nicht gebührende Vorteile», sofern sie vorgängig möglichst präzise sowohl in Hinsicht auf die Leistungen als auch auf die Abgeltung schriftlich vereinbart werden und in einem angemessenen Verhältnis zur Abgeltung stehen [18]. 
Nicht erlaubt ist die Abgeltung von Leistungen, welche eine Fachperson oder Organisation für sich selbst oder in Erfüllung einer gesetzlichen Verpflichtung erbringt, oder wenn sie dafür bereits anderweitig eine Vergütung erhält. Denkbar wäre das, wenn eine Fachperson sich den Aufwand ihrer obligatorischen Weiterund Fortbildung bezahlen lässt, wenn sie eigene Arbeitsabläufe vereinfacht oder wenn die Kosten bereits durch den Lohn oder die tarifarische Vergütung gedeckt sind [19].

Als Beispiele für Gegenleistungen, die - sofern schriftlich vereinbart und in einem angemessenen Verhältnis zur Abgeltung stehend - abgegolten werden können, sind zu nennen: Leistungen beim Einkauf wie die Übernahme von Logistikaufwand, Lagerkosten oder Lagerrisiko, Lehr-, Gutachtens- und Beratungstätigkeit sowie die Durchführung von wissenschaftlichen Studien und klinischen Versuchen, Praxiserfahrungsberichte, wenn sie in einem wissenschaftlich anerkannten Fachmedium publiziert werden, und die Mitwirkung in Beratungsgremien, Workshops oder an Marktforschungen, wenn diese nicht zu Werbezwecken durchgeführt werden [20]. Verhältnismässig ist die Teilnahme an solchen Anlässen aber nur, wenn sie für die Fachperson weniger fordernd ist als die tägliche Arbeit und wenn kein Haftungsrisiko besteht. Andernfalls muss die Höhe der Abgeltung angepasst werden [21].

Die Abgeltung muss mittels separater Zahlung oder Verrechnung erfolgen, die Gegenleistung muss dem Wert der Abgeltung ungefähr entsprechen. Deshalb sind mit einem zusätzlichen Aufwand verbundene Gegenleistungen höher zu bewerten als solche, die bereits im Rahmen der ordentlichen Berufsausübung erbracht werden.

Auf eine schriftliche Vereinbarung kann verzichtet werden, wenn im Rahmen eines Fachgesprächs Verpflegungskosten von höchstens CHF 100 übernommen werden [22].

\section{Rabatte}

Wenn Preisrabatte oder Rückvergütungen keinen Einfluss auf die Wahl der Behandlung haben, gelten sie nicht als «nicht gebührende Vorteile» [23]. Ob dies erfüllt ist, muss im Einzelfall entschieden werden. Rabatte können als Preisrabatte (Fixbetrag oder Prozentsatz des Bruttopreises) oder als Mengenrabatt (Verhältnis zwischen Liefermenge und zu bezahlender Menge) erteilt werden. Auch sind Rückvergütungen möglich.

Unzulässig sind Rabatte, sofern sie dazu führen können, dass ein ungeeignetes verschreibungspflichtiges
Arzneimittel oder ein geeignetes verschreibungspflichtiges Arzneimittel übermässig verschrieben, abgegeben oder angewendet wird, oder wenn dies geschieht, obwohl der Einsatz eines Heilmittels überhaupt nicht angezeigt ist [24].

Naturalrabatte - es werden mehr Produkte geliefert als tatsächlich bezahlt - sind unzulässig [25].

Musterverpackungen dürfen nicht weiterverkauft werden. Ihr Zweck besteht darin, den Fachpersonen zu erlauben, in der Praxis erste Erfahrungen zu sammeln. Darin unterscheiden sie sich von den Naturalrabatten. Ihre Anwendung zum vorgesehenen Zweck ist im Gegensatz zu den Naturalrabatten erlaubt [26].

\section{Transparenz}

Die neuen Transparenzbestimmungen sind beim Einkauf aller Heilmittel anwendbar, und sie beziehen sich auf sämtliche gewährten Rabatte und Rückvergütungen. Diese müssen in den Rechnungen und Geschäftsbüchern sowohl auf Hersteller- und Vertreiber- als auch auf Einkäuferseite eindeutig nachvollziehbar ausgewiesen und auf Verlangen offengelegt werden [27]. Die Offenlegung hat gegenüber dem BAG zu erfolgen, und sie erstreckt sich nur auf die letzte Handelsstufe, also jene Fachpersonen und Organisationen, welche Arzneimittel verschreiben, abgeben, anwenden oder zu diesem Zweck einkaufen [28].

Ausgeschlossen von der Transparenzpflicht sind Heilmittel der Abgabekategorie E sowie Medizinprodukte, welche nach europäischem Recht in die niedrigstschwellige Klasse I fallen, weil sie eher geringe Gesundheitsrisiken bergen [29].

\section{Weitergabe von Vergünstigungen}

Vergünstigungen sind vom Leistungserbringer in der Rechnung aufzuführen und an den Schuldner weiterzugeben, es sei denn, die Vergünstigung fliesse bereits über niedrigere Kosten in die Berechnung der Tarife und Preise der Leistungen ein. In diesen Fällen erübrigt sich ein separates Ausweisen in der Rechnung [30]. Eine nur teilweise Weitergabe von Vergünstigungen kann zwischen den Verbänden der Leistungserbringer und der Versicherer schriftlich vereinbart werden, wobei mehr als 50\% an den Schuldner der Leistung weitergegeben werden müssen [31]. Eine Verbandslösung ist aber nicht zwingend. In der Vereinbarung muss aufgeführt werden, welcher Art die Vergünstigungen sind, in welchem Umfang diese gewährt werden, wie die transparente Dokumentation in Belegen und Rechnungen auszusehen hat, welchem Verwendungszweck nicht weitergeleitete Vergütungen zugeführt werden, 
wozu auch die Verbesserung der Behandlungsqualität gehört, und wie diese nachgewiesen werden muss. In erster Linie werden nicht weitergegebene Mittel zugunsten national ausgerichteter Programme zur Verbesserung der Behandlungsqualität eingesetzt. Leistungserbringer und Versicherer müssen das BAG über entsprechende Vereinbarungen unverzüglich informieren, eine Offenlegung zu diesem Zeitpunkt wird nicht verlangt [32].

In der parlamentarischen Diskussion wurde darauf hingewiesen, dass es durchaus denkbar sei, diese Verträge via Öffentlichkeitsgesetz auf Antrag auch Dritten einsehbar zu machen [33]. Ob einem solchen Antrag stattgegeben wird, muss im Einzelfall entschieden werden. Jedenfalls ist dieser Aspekt bei der Redaktion der entsprechenden Verträge im Auge zu behalten.

\section{Sanktionen}

Primär werden die Bestimmungen zur Integrität und Transparenz im Verwaltungsverfahren vom damit betrauten BAG vollzogen [34]. Dieses kann alle erforderlichen Massnahmen treffen, um den rechtmässigen $\mathrm{Zu}$ stand wiederherzustellen [35].

\section{Die angedrohten Sanktionen sind Freiheits- strafe bis zu drei Jahren oder Geldstrafe.}

Unabhängig davon kann das BAG ein Strafverfahren durchführen bzw. einleiten. Die angedrohten Sanktionen sind Freiheitsstrafe bis zu drei Jahren oder Geldstrafe, wenn eine vorsätzliche oder fahrlässige Verletzung der Integritätsvorschriften geahndet wird. Busse bis zu CHF 50000 wird bei vorsätzlicher Verletzung der Transparenzvorgaben angedroht [36]. Das BAG kann zudem Tätigkeitsverbote, die Einziehung unrechtmässiger Gewinne oder eine Ersatzforderung anordnen [37].

Falls das EDI der Ansicht ist, es komme im Falle der Verletzung von Integritätsregeln eine Freiheitsstrafe in Frage, werden die Akten vom BAG der kantonalen Staatsanwaltschaft zur weiteren Bearbeitung übergeben [38].

Sekretariat

Rechtsdienst FMH

Tel. 0313591111

Fax 0313591112

lex[at]fmh.ch
Literatur

1 Art. 55 Abs. 3, Art. 56 Abs. 2 und 3 HMG

2 https://www.bag.admin.ch/bag/de/home/medizin-undforschung/heilmittel/aktuelle-rechtsetzungsprojekte/ integr-transp-obligation.html

3 Art. 2 lit. a VITH.

4 Erläuterungen des EDI zur Verordnung über Integrität und Transparenz im Heilmittelbereich (VITH) und zur Änderung der Verordnung über die Krankenversicherung (KVV), April 2019, S. 12.

5 Art. 89 HMH i.V.m. Art. 7 Abs. 1 und 2 VStR und Art. 102 Abs. 1 und 4 StGB; Art. 86 Abs. 1 lit. h, Art. 87 Abs. 1 lit. h VITH.

6 Art. 2 lit. b VITH.

7 Art. 55 Abs. 2 lit. a; Art. 3 Abs. 1 VITH.

8 Erläuterungen des EDI zur Verordnung über Integrität und Transparenz im Heilmittelbereich (VITH) und zur Änderung der Verordnung über die Krankenversicherung (KVV), April 2019, S. 13.

9 Art. 3 Abs. 3 VITH.

10 Erläuterungen des EDI zur Verordnung über Integrität und Transparenz im Heilmittelbereich (VITH) und zur Änderung der Verordnung über die Krankenversicherung (KVV), April 2019, S. 14; Art. 55 Abs. 2 lit. b HMG.

11 Art. 4 VITH.

12 Art. 5 VITH

13 Erläuterungen des EDI zur Verordnung über Integrität und Transparenz im Heilmittelbereich (VITH) und zur Änderung der Verordnung über die Krankenversicherung (KVV), April 2019, S. 15.

14 Erläuterungen des EDI zur Verordnung über Integrität und Transparenz im Heilmittelbereich (VITH) und zur Änderung der Verordnung über die Krankenversicherung (KVV), April 2019, S. 16.

15 Art. 6 VITH; Erläuterungen des EDI zur Verordnung über Integrität und Transparenz im Heilmittelbereich (VITH) und zur Änderung der Verordnung über die Krankenversicherung (KVV), April 2019, S. 16.

16 Art. 6 VITH.

17 Art. 6 VITH.

18 Art. 7 VITH.

19 Art. 7 VITH; Erläuterungen des EDI zur Verordnung über Integrität und Transparenz im Heilmittelbereich (VITH) und zur Änderung der Verordnung über die Krankenversicherung (KVV), April 2019, S. $17 f$.

20 Art. 7 VITH

21 Erläuterungen des EDI zur Verordnung über Integrität und Transparenz im Heilmittelbereich (VITH) und zur Änderung der Verordnung über die Krankenversicherung (KVV), April 2019, S. 18.

22 Art. 7 VITH; Erläuterungen des EDI zur Verordnung über Integrität und Transparenz im Heilmittelbereich (VITH) und zur Änderung der Verordnung über die Krankenversicherung (KVV), April 2019, S. 17.

23 Art. 55 Abs. 2 lit. d HMG.

24 Art. 8 VITH.

25 Art. 8 Abs. 2 VITH.

26 Art. 9 VITH

27 Art. 55 Abs. 1 HMG; Art. 10 Abs. 1 VITH.

28 Art. 10 Abs. 1 VITH.

29 Art. 23 Abs. 2, Art. 45 Abs. 3 HMG i.V.m. Art. 1 und 5 MedV; Anhang IX der Richtlinie 93/42/EWG über Medizinprodukte.

30 Art. 42, Art. 56 Abs. 3, Art. 76a KVV.

31 Erläuterungen des EDI zur Verordnung über Integrität und Transparenz im Heilmittelbereich (VITH) und zur Änderung der Verordnung über die Krankenversicherung (KVV), April 2019, S. 21.

32 Art. 76b KVV.

33 https://www.parlament.ch/de/ratsbetrieb/amtliches-bulletin/amtliches-bulletin-die-verhandlungen?SubjectId=36921\#votum3; https://www.admin.ch/opc/de/classified-compilation/20022540/ index.html

34 Art. 84 Abs. 1 HMG

35 Art. 66 Abs. 1 i.V.m. Art. 82 Abs. 1 HMG

36 Art. 86 Abs. 1 lit. h HMG; Art. 87 Abs. 1 lit. h HMG.

37 Art. 2 VStrR i.V.m. Art. 67ff. StGB.

38 Art. 21 Abs. 1, Art. 73 Abs. 1 VStrR.

39 Art. 322ter ff. StGB. 